\title{
Potts model on the Sierpínski gasket: A transfer-matrix approach
}

\author{
R. F. S. Andrade \\ Instituto de Fisica, Universidade Federal da Bahia, 40210-340 Salvador, Brazil
}

(Received 2 August 1993)

\begin{abstract}
The $q$-states Potts model on the Sierpínski gasket with ferromagnetic and antiferromagnetic interactions is analyzed with use of the transfer-matrix method. It leads to a set of four coupled maps for the free energy and correlation length. Attention is focused on the behavior of the residual entropy, when 1, 2 , or 3 bonds within a unit cell is antiferromagnetic. In the isotropic case, with $q=4$, the value for $s_{0}$ is larger than that of the triangular lattice. The isotropic ferromagnetic model shows an unusual sharp peak in the specific heat in the limit of large $q$.
\end{abstract}

\section{INTRODUCTION}

The thermodynamic properties of spin models on fractal lattices have been intensively investigated in the last decade. It was very quickly recognized that models on fractals with finite order of ramification do not present critical behavior at nonzero temperature. ${ }^{1,2}$ Yet such models are very interesting since it is possible to obtain exact results on their thermodynamic properties, some of which are rather nontrivial.

For these fractals, the methods which take advantage of their scale invariance lead to exact recursion relations linking a given thermodynamic function of two subsequent generations in the construction of the fractal. This is true both for the renormalization group (RG), ${ }^{1-3}$ and also, as it has been more recently explored, for the partition function ${ }^{4-6}$ and/or transfer matrices (TM). ${ }^{7-10}$

In this paper we explore some results for the Potts model on the Sierpínski gasket (SG). For this purpose we use the TM approach we have recently introduced ${ }^{8}$ to obtain, after the numerical iteration of a set of recurrence maps, the free energy and correlation length of the model. We consider that bonds of two different kinds are present in the model, and our main attention is focused on the residual entropies, when we have 1,2 , and 3 antiferromagnetic (AF) bonds in each unit triangle. Similar results for the Ising model have been recently explored in detail. ${ }^{8}$ As we will show, the Potts model presents a richer behavior than the Ising model, since the degenerate situations (e.g., one or three AF bonds in a unit triangle) will be lifted when $q \geq 3$. Besides the discussion of the different AF situations we also briefly comment on the most interesting features when all bonds are ferromagnetic.

Recursion relations for the partition function of the Potts model on the SG have been published recently. ${ }^{6}$ This approach is based on very similar ideas as ours, so that our and their maps should be brought into connection. However, the results we present here have not been discussed therein, since the authors have used the Potts model for the derivation of relevant aspects of cluster statistics (the $q \rightarrow 1$ limit).

\section{RECURRENCE MAPS}

The TM recursion relations for any nearest-neighbor spin model on the SG were presented in Ref. 8. They can be written in terms of two matrices, $A_{n}$ and $U_{n}$, respectively of order $q \times q$ and $q \times q^{2}$, where $q$ refers to the number of distinct states that the spin variable may assume, and read

$$
\begin{aligned}
& A_{n+1}=U_{n}\left(A_{n} * I\right) U_{n}^{t}, \\
& \left(U_{n+1}\right)_{i, j k}=\sum_{p, q, r}\left(U_{n}\right)_{i, p q}\left(U_{n}\right)_{j, q r}\left(U_{n}\right)_{k, p r} .
\end{aligned}
$$

In (1), $I, t$, and $*$ indicate, respectively, the identity $q \times q$ matrix, the transpose of the matrix $U$, and the direct (Kronecker) product between the matrices $A_{n}$ and $I$. In (2) we use the lexicographic order for the column label of the matrix $U$. The matrices of the first generation, which will fix the initial conditions for the maps to be iterated, are given by

$$
\begin{aligned}
& \left(A_{1}\right)_{i j}=A_{i j}^{2} C_{i j}, \\
& \left(U_{1}\right)_{i, j k}=A_{i j} A_{j k} C_{i k},
\end{aligned}
$$

where the matrices $A$ and $C$ describe the interaction between two neighboring spin variables, say $s^{1}$ and $s^{2}$, and are expressed in terms of the coupling constants $K=\beta J$ and $K^{\prime}=\beta J^{\prime}$ (we suppose no magnetic field is present) as

$$
\begin{aligned}
& A_{i j}=\exp \left[K \delta\left(s_{i}^{1}, s_{j}^{2}\right)\right], \quad i, j=1, \ldots, q, \\
& C_{i j}=\exp \left[K^{\prime} \delta\left(s_{i}{ }^{1}, s_{j}^{2}\right)\right], \quad i, j=1, \ldots, q .
\end{aligned}
$$

The basic cell of the model contains two $J$ and one $J^{\prime}$ coupling, corresponding, respectively, to the two tilted and one horizontal bonds of the model (see Fig. 1).

It turns out that the matrix $A_{1}$ (and also all subsequent matrices $A_{n}, n \geq 1$ ) have only two independent matrix elements, $a_{1}$ and $b_{1}$, placed, respectively, at the diagonal and off-diagonal positions. The eigenvalues of $A_{1}$ are

$\eta_{1}=a_{1}+(q-1) b_{1}=e^{2 K+K^{\prime}}+(q-1)\left(2 e^{K}+e^{K^{\prime}}+q-2\right)$, 


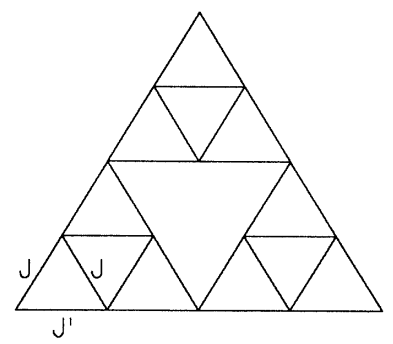

FIG. 1. The second generation of the construction of the Sierpínski gasket with two types of bonds.

$\varepsilon_{1}=a_{1}-b_{1}=e^{2 K+K^{\prime}}+(q-1) e^{K^{\prime}}-e^{K}+2-q$,

where $\varepsilon_{1}$ is $(q-1)$-fold degenerate. If we write $U_{1}$ in the basis where $A_{1}$ is diagonal, i.e., if we consider the matrix $\widetilde{U}=\gamma^{-1} U_{1}\left(\gamma^{*} \gamma\right)$, where $\gamma$ is the matrix of eigenvectors of $A_{1}$, we note that, besides $\eta_{1}$ and $\varepsilon_{1}$, it contains only two new elements:

$$
\varphi_{1}=e^{2 K+K^{\prime}}-e^{K^{\prime}}+(q-2) e^{K}+2-q,
$$

$$
\mu_{1}=e^{2 K+K^{\prime}}-2 e^{K^{\prime}}-e^{K}+2 .
$$

This set of four variables describes the whole thermodynamic behavior of the model. However the recurrence maps, which follow from Eqs. (1) and (2), can be written in a more suitable form for the purpose of the numerical evaluation of the properties of the model by introducing new variables:

$$
\begin{aligned}
& f_{n}=-T \ln \left[\eta_{n}+(q-1) \varepsilon_{n}\right] / N_{n}, \\
& \xi_{n}=M_{n} / \ln \left[\eta_{n} / \varepsilon_{n}\right], \\
& \xi_{n}=M_{n} / \ln \left[\eta_{n} / \varphi_{n}\right], \\
& \chi_{n}=M_{n} / \ln \left[\eta_{n} / \mu_{n}\right],
\end{aligned}
$$

where $f_{n}$ is the free-energy per spin and $\xi_{n}$ the correlation length. $\zeta_{n}$ and $\chi_{n}$ are auxiliary variables, defined similarly as $\xi_{n}$, but they do not have any physical meaning. $N_{n}=\left(3^{n}+3\right) / 2$ is the number of sites in the $n$th generation of the construction of the fractal and $M_{n}=2^{n}$ is the shortest distance between the two spins placed in the outermost corners in the $n$th generation of the fractal. The recurrence maps which follows from (1) and (2) read

$$
\begin{aligned}
& f_{n+1}=3 \frac{N_{n}}{N_{n+1}} f_{n}-\frac{T}{N_{n+1}} \ln \frac{1+(q-1) x_{n}^{2}+2(q-1) x_{n} y_{n}^{2}+(q-1)(q-2) x_{n} z_{n}^{2}}{q\left[1+(q-1) x_{n}\right]^{3}}, \\
& \xi_{n+1}=\xi_{n}\left[1+\frac{\xi_{n}}{2 M_{n}} \ln \frac{1+(q-1) x_{n} y_{n}^{2}}{1+x_{n}^{-1} y_{n}^{2}+(q-2) x_{n}^{-1} z_{n}^{2}}\right]^{-1}, \\
& \xi_{n+1}=\xi_{n}\left[1+\frac{\zeta_{n}}{2 M_{n}} \ln \frac{1+(q-1) x_{n} y_{n}^{2}}{1+x_{n}+(q-2) y_{n}^{-1} z_{n}^{2}}\right]^{-1}, \\
& \chi_{n+1}=2 \chi_{n}\left[1+\frac{2 \chi_{n}}{\zeta_{n}}+\frac{\xi_{n}}{M_{n}} \ln \frac{1+(q-1) x_{n} y_{n}^{2}}{1+2 x_{n} y_{n}^{-1}+(q-3) y_{n}^{-2} z_{n}^{2}}\right]^{-1},
\end{aligned}
$$

where $x_{n}=\varepsilon_{n} / \eta_{n}, y_{n}=\varphi_{n} / \eta_{n}, z_{n}=\mu_{n} / \eta_{n}$. We observe that when $q=2$, the map for $\chi_{n}$ is decoupled from the other three, and also that, in the isotropic situation, $K=K^{\prime}$, the $\left\{\zeta_{n}\right\}$ and $\left\{\xi_{n}\right\}$ are identical.

\section{RESULTS}

\section{A. Ferromagnetic situation $J=J^{\prime}>0$}

When all bonds are ferromagnetic, the most unusual feature we have detected refers to the linear dependence of the maxima of the specific heat with respect to $q$ $\left(c_{\max } \sim q / 2\right)$. Figure 2 shows that the slope of the curve is also very large, leading to very thin peaks when $q$ increases. This result contrasts with that for the linear chain, where $c_{\max }$ grows only with $[\ln (q)]^{2} / 4$ and the slope of the curve is small. Though the curves are smooth everywhere, this indicates that the geometry of the SG brings the models much closer to a criticality than the linear chain.

The second relevant feature refers to the dependence of $\xi$ with $T$ as $T \rightarrow 0$. Like the Ising model, ${ }^{10,11}$ we also find that $\xi \sim \exp [\exp (1 / T)]$. This behavior is observed for any value of $q$, also when we extend $(15)-(18)$ to real numbers and even when $q<1$. In this last case we observe that, much as it happens with the linear chain, the results for the free energy become nonphysical (e.g. the specific heat becomes negative). Nevertheless, the correlation is still a well defined quantity, whose behavior is the one quoted above.

$$
\text { SIERPINSKI; POTTS; } J=1 ; \quad J=1
$$

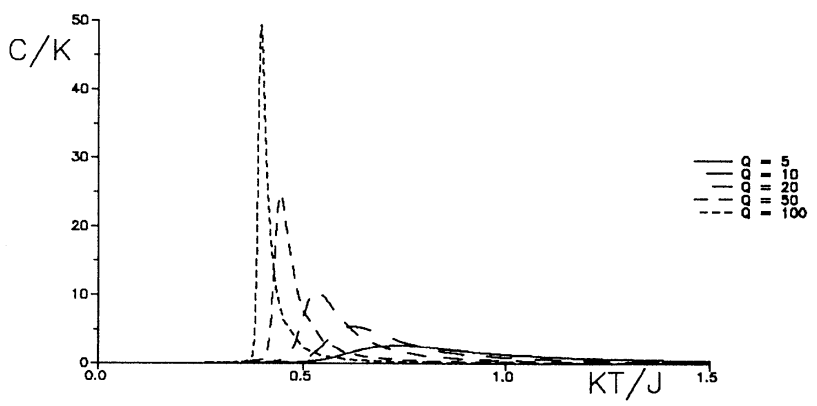

FIG. 2. The specific heat in the ferromagnetic case for different values of $g$. 


\section{B. 3 AF bonds: $J=J^{\prime}<0$}

We will now focus our discussion on the residual entropy $s_{0}(q)=s(T=0, q)$ of the model. We recall that the Potts model on the SG, as in the triangular lattice, is nonfrustrated for $q \geq 3$. However the ground state may be highly degenerate, which explains the presence of a residual entropy.

When $q=3$ there is no macroscopic degeneracy of the ground state, which is confirmed by our numerical evaluation when we find $s_{0}(3)=0$. Long distance order settles in, but not as fast as in the ferromagnetic case, since now we find $\xi \sim \exp (1 / T)$.

The value for $s_{0}(q=4)$ can be exactly evaluated from the maps (15)-(18). For this particular value the map (15) decouples from the other three already in the second iteration, making it possible to find its limit value when $n \rightarrow \infty$. However, this does not happen for the other values of $q$, when we must compute numerically the value of $s_{0}$. The value we obtained for $s_{0}(4)$ is larger than the well-known value for the exact residual entropy for the same model on the triangular lattice: ${ }^{12,13}$

$$
\begin{aligned}
& s_{0}(4) / k=\frac{2}{9} \ln 24 \cong 0.706234, \\
& s_{0}^{t}(4) / k=\ln (1.46099) \cong 0.37911 .
\end{aligned}
$$

The physical reasons for this result must be the same used to explain the similar situation observed for the $q=2$ (Ising) model: ${ }^{4,5,14}$ the removal of certain welldefined bonds from the triangular lattice leading to the SG causes an increase in the degeneracy of the system. On the other hand, the random bond diluted spin glass on the triangular lattice should present a lower residual entropy.

In Fig. 3 we draw $s_{0}(q) / s_{\infty}(q) \times q$, where $s_{\infty}(q)$ $=k \ln (q)$ is the high-temperature limit of the entropy. The curve shows that $s_{0}(q) \sim s_{\infty}(q)$ as $q$ increases. In this limit the ratio of the number of possible configurations in the low- to the high-temperature limit becomes closer and closer (actually $1-1 / q$ per bond). The extension of the results for real values of $q$ in this figure is limited to $q \geq 3$, since for $q<3$ the maps (15) $-(18)$ lead to nonphysical results. The $q=2$ is the

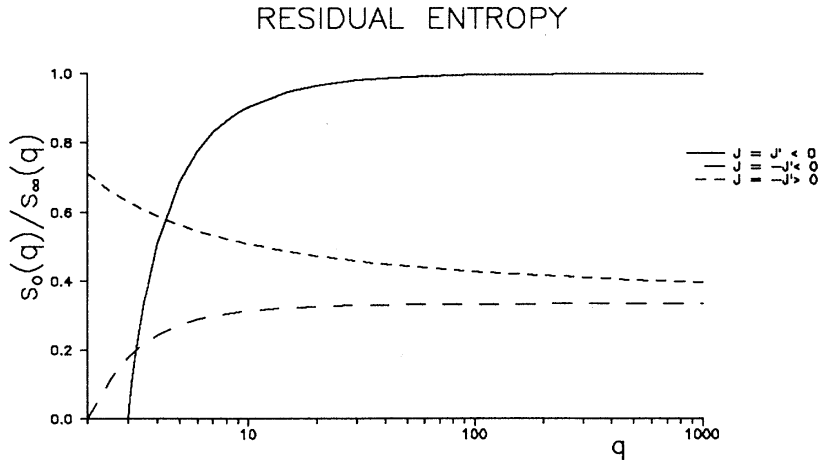

FIG. 3. $s_{0}(q) / s_{\text {in }} f(q)$ as function of $q$ when 1,2 , and $3 \mathrm{AF}$ bonds are present. For the 3-AF-bond case the residual entropy is not defined for $2<q<3$. only isolated singular point in the $(0,3)$ interval where the results are well behaved.

We finally mention that the correlation length is bounded as $T \rightarrow 0$ for all values of $q$ where $s_{0} \neq 0$. The low-temperature value of $\xi$ increases very slowly when $q \rightarrow 3^{+}$.

\section{2 AF bonds: $J=-J^{\prime}<0$}

This is again a nonfrustrated situation, which becomes degenerate with the pure ferromagnetic case when $q=2$. Therefore at this $q$ value it is characterized by the absence of residual entropy and strong divergence of the correlation length. For all other integer values of $q$ we find a nonzero residual entropy. In such cases the two spins of the unit cell (triangle) linked by the $F$ bond must be in the same state, while the third one must be in one of the other $(q-1)$ states, giving rise to a macroscopic degeneracy of the ground state.

In the discussion of the low-temperature behavior of $\xi$ we observe that it strongly depends on the direction. Since the horizontal bond is ferromagnetic, all spins in a same row along this direction will be in one same state. On the other hand, the existence of a multiplicity of energetically equivalent configuration for those spins linked by tilted bonds, makes the correlation in such directions decay rapidly. The numerical evaluation of $\xi$ shows that, when $q \neq 2$, it diverges with $\exp (1 / T)$. Due to the reasons given before, and to the way the transfer matrices are defined, this result refers only to the correlation length in the horizontal direction.

The extension of the results to noninteger values of $q$ is restricted to the interval $q>2$. As in what happens with the AF situation, this limit is dictated by the value of $q$ where the residual entropy vanishes. Figure 3 shows that the residual entropy in the limit of large $q$ approaches $s_{\infty} / 3$, showing that the ground state is far less degenerate than in the AF situation.

\section{1 AF bond: $J=-J^{\prime}>0$}

This is the only situation discussed here where frustration occurs for all integer values of $q \geq 2$. When $q=2$ this situation is degenerated with the isotropic AF one. Unlike the two cases presented before, the residual entropy is due to the multiplicity of different places where the frustrated bond may be placed within each triangle. From Fig. 3 we note that now $s_{0}(q) / s_{\infty}(q)$ decreases with $q$, but, in the limit of large $q$, it approaches the same value as in the situation discussed before: $s_{0}(q) / s_{\infty}(q) \sim \frac{1}{3} \ln (q)$.

As what happens in the 3-AF-bond situation, it has been also possible to obtain here, for the unique value $q=3$, the exact value of the residual entropy $s_{0} / k=\frac{1}{9} \ln (507)$.

The correlation length remains bounded as $T \rightarrow 0$, and it is easy to see that, despite the asymmetry in the character of the horizontal and tilted bonds, $\xi$ must be the same in all directions.

The extension of the results to noninteger values of $q$ cannot be limited by a value where the residual entropy vanishes. Nevertheless the corresponding curve in Fig. 3 
also stops when $q=2$. For $q<2$ our results indicate that there exists a temperature interval around $T=0$ where the entropy, after going through a local minimum, starts increasing as $T$ decreases. This temperature interval increases when we decrease $q$.

\section{CONCLUSIONS}

In this paper we have discussed the behavior of the Potts model on the SG for different choices of $F$ and AF bonds. In the $F$ case we have shown that as $q$ increases, the curves of specific heat become very sharp, with very large maxima. This behavior is very far from that typical for the linear chain. This shows once more that, among the finite ramified planar fractal lattices, the SG has indeed the richest set of peculiar features which are nontypical for 1- $d$ systems. The residual entropy has been discussed for the cases where 1, 2, and $3 \mathrm{AF}$ bonds are present in each unit cell. In the first case it is due to the presence of one frustrated bond per cell, while for 2 and 3 AF bonds it comes from the multiplicity of energetically equivalent states allowed in the Potts model. The results were summarized in Fig. 3, which shows the behavior of $s_{0}(q) / s_{\infty}(q)$ with $q$ for the three situations. Our results for $q=4$ show that, like in the $q=2$ case, the residual entropy per spin in the SG is higher than that in the $q=4$ triangular lattice, and it is natural to conjecture that the same will be valid for any value of $q$.

The maps which describe the system were obtained with the help from computer algebra programs from the TM recursion relation, which is valid for any model in the SG. This supports the convenience of using this method, since one set of TM relations describe any model on the same fractal. We stress that the maps (15)-(18) are valid for any value of $J$ and $J^{\prime}$, not being limited to the situations $|J|=\left|J^{\prime}\right|$ discussed in this paper.
${ }^{1}$ Y. Gefen, B. Mandelbrot, and A. Aharony, Phys. Rev. Lett. 45, 955 (1980).

${ }^{2}$ Y. Gefen, A. Aharony, and B. Mandelbrot, J. Phys. A 16, 1267 (1983).

${ }^{3}$ D. Dhar, J. Math. Phys. 19, 5 (1978).

${ }^{4}$ R. B. Stinchcombe, Physica D 38, 345 (1989).

${ }^{5}$ R. B. Stinchcombe, Phys. Rev. B 41, 2510 (1990).

${ }^{6}$ Z. Borjan, M. Knezevic, and S. Milosevic, Phys. Rev. B 47, 144 (1993).

${ }^{7}$ R. F. S. Andrade and S. A. Salinas, J. Phys. A 17, 1665 (1984).
${ }^{8}$ R. F. S. Andrade, Physica A 193, 29 (1993).

${ }^{9}$ P. M. Adler, in The Fractal Approach to Heterogeneous Chemistry, edited by D. Avnir (Wiley, Chichester, 1989).

${ }^{10}$ S. H. Liu, Phys. Rev. B 32, 5804 (1985).

${ }^{11}$ Y. Gefen, A. Aharony, Y. Shapir, and B. Mandelbrot, J. Phys. A 17, 435 (1984).

${ }^{12}$ R. J. Baxter, J. Math. Phys. 11, 784 (1970).

${ }^{13}$ F. Y. Wu, Rev. Mod. Phys. 54, 235 (1982).

${ }^{14}$ M. P. Grillon and F. G. Brady-Moreira, Phys. Lett. A 142, 22 (1989). 